\title{
Modeling Protein-Based 3-D Memory in SPICE
}

\author{
Ronald W. Brower, Robert L. Ewing, Andrew J. Brower \\ Air Force Research Laboratory \\ Wright Patterson Air Force Base, Ohio 45433 \\ ronald.brower@wpafb.af.mil, robert.ewing $a$,wpafb.af.mil and andrew.brower@wpafb.af.mil
}

\begin{abstract}
The Simulation Program for Integrated Circuit Engineering (SPICE) has been used successfully for many years to aid semiconductor circuit design. SPICE represents circuits as networks of basic devices with interacting parameters (current, voltage, etc.), and then models these interactions as systems of partial differential equations. Biological systems are roughly similar, consisting of networks of interacting biochemical cycles whose resulting chemical concentrations can also be modeled by partial differential equations. In the present work, we use SPICE to model an optically-accessed, protein-based, 3-D memory. Modeling the protein's photochemical effects in SPICE allows us to include, within the same model, the supporting electronics and optics needed to properly cycle the memory and interface it to the outside world.
\end{abstract}

\section{INTRODUCTION}

For about 30 years, the Simulation Program with Integrated Circuit Emphasis (SPICE) has been used for analyzing and simulating integrated circuit (IC) designs [1]. Since the parameters of IC operation are governed by systems of differential equations, SPICE in effect finds a numerical solution to these differential equations. This suggests that the SPICE engine can be applied to other domains wherein differential equations govern.

Consider the field of biology. Biological processes can be described as networks of interacting chemical reactions that compete for reactants, and produce products that may catalyze or inhibit other reactions. Thus a biological process can often be modeled as a system of chemical rate equations, whose solution can be used to analyze or predict biological behavior. [2] [3] [4] [5] [6]

At the Air Force Research Laboratory, we are also interested in using SPICE to model biochemical processes. Whereas other researchers have used SPICE for one-time analysis of these processes, we aim to use SPICE as a true design tool: One would have a library of validated models that can then be inserted into larger, composite designs to be simulated and evaluated. Such a tool would support, for example, the Cell-Like Entity project of our Human Effectiveness Directorate, which includes an initiative to build computation elements from sub-cellular biomaterials [7].
We are currently using SPICE to model a protein-based 3-D optical memory made from bacteriorhodopsin. Previously, we reported our work on a simple, three-state model that used a non-linear current source ("B" device) to mimic the action of the read, write and erase lasers [10]. In our present work, we have built a more accurate nine-state model of the bacteriorhodopsin photo response, and have replaced the $\mathrm{B}$ devices with models of commercial lasers implemented as vertical-cavity surface-emitting lasers (VCSELs).

\section{BACKGROUND}

Schmidt, Stuart, Singh and Birge provide a good description of the origins of bacteriorhodopsin and its potential as a 3-D memory [8]. We provide a brief summary here.

Bacteriorhodopsin (BR) is a protein used by the saltmarsh-dwelling bacterium, Halobacterium salinarum, for a primitive form of photosynthesis. This organism exploits BR's ability to absorb light and pump a proton across the molecule. A membrane of aligned BR molecules consequently builds up a significant charge gradient which the organism taps for energy. BR also has a two-photon process - not used in nature-which allows it to serve as an optically accessed three-dimensional memory.

As will be seen in Fig. 2, BR's photo cycle has several energetic states. The ground state is called simply "BR." A green laser will excite the material from state $B R$, through several intermediate states, until the " $\mathrm{O}$ " state is reached after about 2 milliseconds. Once the green laser ceases, in the absence of any other excitation, the material will eventually decay thermally back to the BR state. However, while still in the $\mathrm{O}$ state, a red wavelength can excite the material to the "P" state, which then transforms thermally to the "Q" state. Q is stable for several years, or until a subsequent blue erase pulse returns the material to state BR. For memory operation, the BR state represents binary ZERO and the P-Q combination represents binary ONE. The material is naturally at ZERO. Writing is accomplished by promoting certain locales to ONE.

This green-red sequence is critical to the 3-D writing process (Fig. 1). A red pulse cannot perform a write unless it follows green closely in time. In operation, a green "paging" 
laser illuminates a slice (page) of the 3-D BR bulk, transforming that page to the $\mathrm{O}$ state. Thus this page, and only this page, is prepared for writing. An array of orthogonally placed red lasers then illuminates the voxels to be written. Outside the selected page, the red is ineffective. Within the selected page, the red-illuminated bits are transformed to PQ.

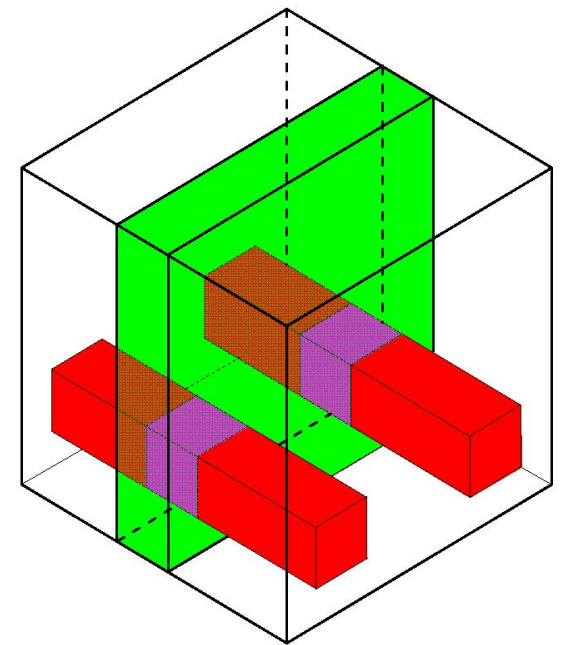

Figure 1. 3-D writing scheme for bacteriorhodopsin.

Reading is accomplished with low-level red light. The level is too low for effective writing, but nevertheless is absorbed by the ZERO voxels. The ONE voxels, having already absorbed red, pass it through. Hence, to a photo detector, a ZERO appears dark and a ONE appears bright.

Finally, for erasing, a blue frequency accomplishes a global erase, returning all $\mathrm{P}$ and $\mathrm{Q}$ material back to $\mathrm{BR}$.

\section{PREVIOUS WORK}

Researchers at the Medical College of Virginia have used SPICE to model various biosynthesis reactions [2] [3] [4] [5] [6]. Their method represents the concentration of a biochemical species as the charge on a capacitor, using one capacitor per species. As the biochemical reaction evolves, changing species concentrations are directly modeled by the charging and discharging of those capacitors. In conventional SPICE usage, an ammeter can be simulated by a controlled voltage ( $\mathrm{V}$ device) set to zero volts. The same technique is used to simulate flow meters for each reactant species. The chemical kinetics and feedback mechanisms (the way species interact to support or inhibit reactions) are directly programmed into controlled sources. The voltagecontrolled current source $(\mathrm{G})$ models a reaction rate (current) controlled by species concentration (voltage). Similarly, the current-controlled current source $(\mathrm{F})$, models one species flow being controlled by another. An important departure from conventional SPICE usage involves the treatment of currents. SPICE assumes current flow consisting of only one species, electrons. For biochemical modeling, the flow of separate species must be kept distinct. This is accomplished by maintaining separate subnetworks for each reactant, with the necessary coupling achieved by the controlled current or voltage sources.

SPICE has also been used to model mixed domain electrical/optical systems. Ytterdal, et al., modeled a smart pixel array by representing free-space optical interconnects as an attenuation factor [9]. Deng, et al., used SPICE to perform electronic/photonic simulation of a semiconductor laser communicating with a photo detector diode, with an intervening optical waveguide modeled as a transmission line [10]. Their work also included a novel thermal subcircuit to account for heating effects.

\section{OUR RESEARCH GOALS}

Previous efforts to use SPICE to model biochemical processes have stressed analysis over design. Researchers have been interested in understanding the underlying chemical kinetics as opposed to creating design models of bio-components to incorporate into systems. We intend to create SPICE models of bio-components to support mixed domain design.

Now mixed domain SPICE modeling is not new. SPICE has been used to model mixed electronic/photonic systems, as was shown in [9] and [10]. However, we are unaware of any mixed domain modeling that includes chemical, biochemical, or bio-photonic effects together with conventional electronics in the same system model.

At AFRL, our goal is to conduct mixed domain modeling of hybrid systems using a SPICE library of bio-memories, bio-gates, and so forth. This will enable us to support several areas of bio-computation research, such as 3-D and holographic memory, protein-based sensors, and AFRL's Cell-Like Entity (CLE) program which seeks to build basic computational structures from subcellular bio-components [7].

For the near term, we are concentrating our efforts on modeling and simulating the optically-activated, proteinbased, three-dimensional memory.

\section{BACTERIORHODPSIN MEMORY MODELING}

Previously, we presented a simplified model of the BR photo response that included only three states: $\mathrm{BR}, \mathrm{O}$ and a lumped PQ [11]. Now we present a more accurate nine state model of the BR photo response. These states are displayed in the photocycle diagram of Figure 2. In the diagram, the colored arrows indicate the light-enabled transitions. The color of the arrow approximates the wavelength that causes the transition. The black arrows indicate thermal transitions. The major thermal transitions of interest are emboldened, though competing reverse transitions are also present and must be modeled. Note that the paging laser only converts $\mathrm{BR}$ to $\mathrm{K}$, and $\mathrm{O}$ is achieved only after a series of subsequent thermal transitions. $\mathrm{O}$ is not permanent, decaying thermally back to BR unless a red pulse is received. 


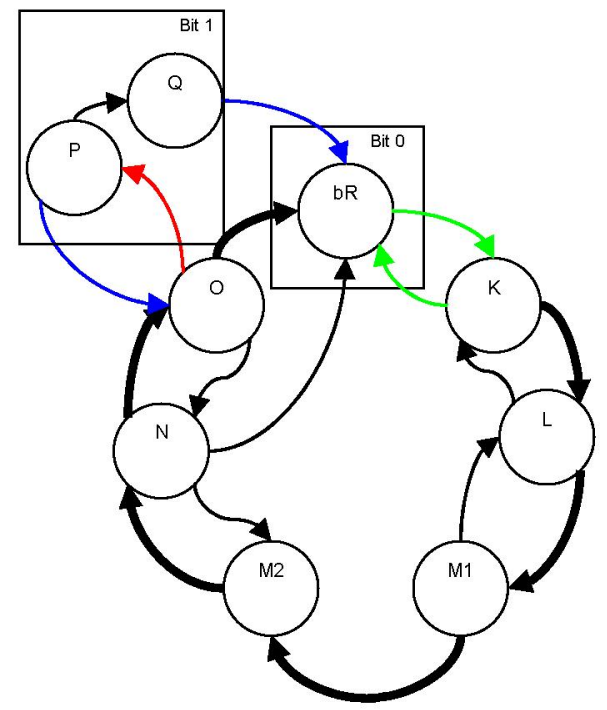

Figure 2. Bacteriorhodopsin photo cycle.

We model the population of molecules in each state by nine capacitors each charged to a level between 0 and 1 volt, indicating the percentage of molecules in each respective state. Initially, the BR capacitor is charged to 1 volt, with all others initialized to 0 . During a state transition, current flows from one capacitor to another, charging the target state at the expense of the previous state. At any instant of time, the total voltage across all capacitors must be 1 volt.

Each thermal decay has a characteristic rate constant governed by an exponential law. This decay has an exact analogy with an $\mathrm{RC}$ circuit, wherein resistor $\mathrm{R}$ drains capacitor $\mathrm{C}$ exponentially, with a rate constant equal to the $\mathrm{RC}$ product. For each thermal transition, we therefore connect a draining resistor across the losing state capacitor with resistance selected to achieve the desired rate constant. The draining current flows through a zero volt dc source (ammeter) such that the drain current can be measured. This current is then added as a term to a controlled current source ("B" device). This B device then charges the target state capacitor at the same rate that the source capacitor discharges. This coupling of charging/discharging currents is shown as the dotted line in Fig. 3.

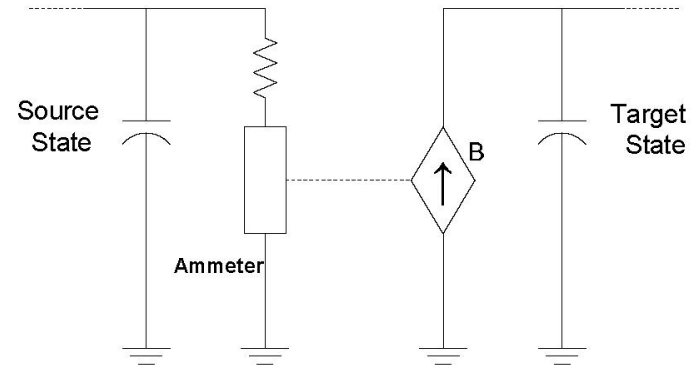

Figure 3. Thermal transition via RC decay.

Fig. 4 gives the basic circuit scheme which we apply again and again for all states. Each state (in this case state
"L") has a single state capacitor. For each outgoing thermal transition we provide a draining resistor selected for the appropriate RC rate constant. Each resistor is accompanied, in series, by an ammeter to measure the draining current. Each state also has a B device to recharge it. For each incoming transition, this B device taps the appropriate draining current from the source state(s), and then charges the target state consistent with the discharge of the source state(s). For the $\mathrm{L}$ state there are two incoming thermal arrows, and hence two contributing current to charge $\mathrm{C}_{\mathrm{L}}$. We also give the corresponding SPICE code in the figure. Note the values of the resistors $\mathrm{R}_{\mathrm{LK}}$ and $\mathrm{R}_{\mathrm{LM} 1}$ which, when multiplied by $\mathrm{C}_{\mathrm{L}}$, give rate constants of $1.54 \mu \mathrm{s}$ and $20.4 \mu \mathrm{s}$ for the $\mathrm{L} \rightarrow \mathrm{K}$ and $\mathrm{L} \rightarrow \mathrm{M} 1$ transitions, respectively.

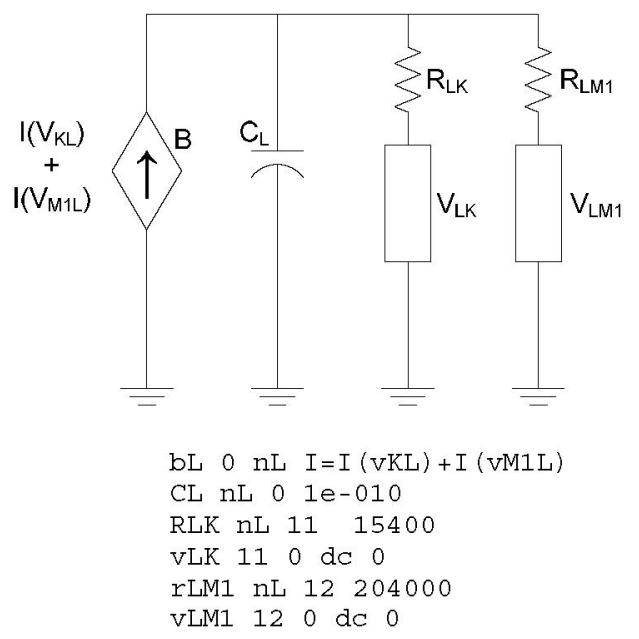

Figure 4. Charging and discharging circuit for state $\mathrm{L}$.

The light-enabled transitions (colored arrows) are handled as follows: First, we model the lasers as semiconductor-based VCSELs (vertical-cavity, surface emitting lasers). We use SPICE VCSEL models available from Honeywell [12]. These laser models are driven by an input current and produce an output current to simulate the produced photons. This current is added to, and subtracted from, the charging B devices of the gaining and losing states, respectively.

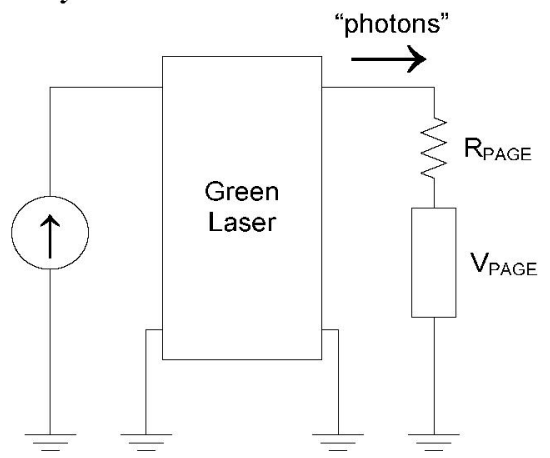

Figure 5. Green, or "paging" laser circuit. 
Consider the green-enabled paging transition $\mathrm{BR} \rightarrow \mathrm{K}$. In the paging laser's circuit, the "photon" flux is measured by ammeter $V_{\text {PAGE}}$. The current $I\left(V_{\text {PAGE }}\right)$ is therefore added as a term in the charging circuits for $\mathrm{C}_{\mathrm{K}}$, and subtracted from that of $\mathrm{C}_{\mathrm{BR}}$. This represents the conversion of $\mathrm{BR}$ to $\mathrm{K}$. However, the $\mathrm{BR} \rightarrow \mathrm{K}$ conversion must slow over time, as the population in BR becomes depleted. In fact, the conversion rate will be directly proportional to the population of $\mathrm{BR}$ remaining. To model this, the current, $\mathrm{I}\left(\mathrm{V}_{\mathrm{PAGE}}\right)$, must be attenuated by multiplying it by the voltage across $\mathrm{C}_{\mathrm{BR}}$. This slows the conversion rate to zero as the BR gets depleted, and prevents $\mathrm{V}\left(\mathrm{C}_{\mathrm{BR}}\right)$ from descending below zero, which would denote negative population. Finally, this current must be further scaled to match the desired rate constant for the conversion. In this case, given the current output of the VCSEL model, a factor of 19.02 yields the desired rate constant of 3.15 ns. Thus the term " $19.02 * \mathrm{I}\left(\mathrm{V}_{\mathrm{PAGE}}\right) * \mathrm{~V}\left(\mathrm{C}_{\mathrm{BR}}\right)$ " is added to the $B$ source charging $C_{K}$ and subtracted from the $B$ source charging $C_{B R}$.

\section{RESULTS}

Presently, we have built the nine-state SPICE model with green, red and blue VCSEL lasers, and have performed simulations of the paging, writing and erasing portions of the memory cycle.

Fig. 6 shows the simulation results for the paging operation. One can see the quick consumption of BR (red trace) by $\mathrm{K}$ (green trace). We see that the $50 \%$ crossing of the $\mathrm{BR}$ and $\mathrm{K}$ levels occurs in about 2 nanoseconds, as expected from descriptions in the literature. After the initial buildup of $\mathrm{K}$, the $\mathrm{K}$ state then gradually depletes, losing population while the subsequent states $\mathrm{L}$ through $\mathrm{O}$ gain via the thermal transitions.

Fig. 7 shows the simulation results for the red laser enabled write operation. The $\mathrm{O}$ state (red trace) does not reach high levels due to competing thermal transitions forever attempting to drain it. However, the conversion of $\mathrm{O}$ to $\mathrm{P}$ is one-way. Therefore, once writing commences, $\mathrm{P}$ immediately consumes whatever $\mathrm{O}$ is produced, and continues to build over time. When the red laser ceases, the $\mathrm{P}$ population maintains it level. Only the $\mathrm{P} \rightarrow \mathrm{Q}$ transition can drain it, and since this transition takes "minutes" (we use 100 seconds for the $\mathrm{P} \rightarrow \mathrm{Q}$ rate constant) we do not see significant production of $\mathrm{Q}$ or consumption of $\mathrm{P}$ in our simulations thus far.

Fig. 8 shows the simulation results for the erase operation. Now erasure converts Q directly to BR, however we do not produce significant $Q$ in our short simulation times. Therefore, our major erase action converts $P$ back to $O$ first, and then this $O$ population decays thermally back to $\mathrm{BR}$. Hence, when $\mathrm{P}$ (green trace) is quickly erased, we see a brief surge of $\mathrm{O}$ (red trace) which then quickly decays, as the material returns thermally to $\mathrm{BR}$.

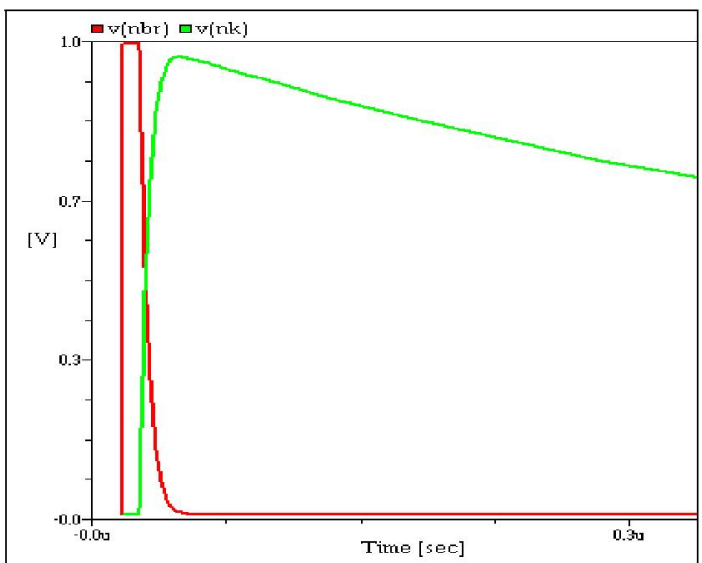

Figure 6. Paging operation. BR level in red (the quickly declining curve). $\mathrm{K}$ in green (slowly declining).

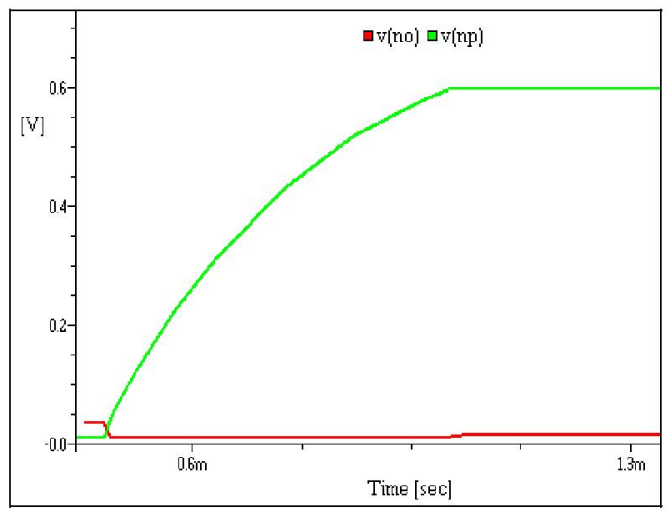

Figure 7. Write operation. O in red (lower curve). P in green (upper curve).

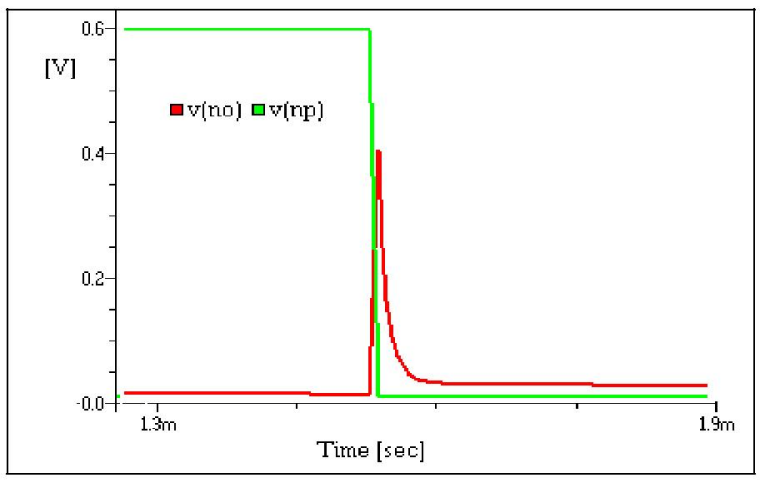

Figure 8. Erase operation. P in green (sharp decline). O in red (brief spike)

\section{CONCLUSIONS}

We now have a model that yields good writing and erasing behavior whose timing is consistent with descriptions in the literature. For each transition, we have used published time constants to the greatest extent possible. Unfortunately, the published constants sometimes differ, are only stated vaguely, or are even missing entirely. (We have no time constant for $\mathrm{O} \rightarrow \mathrm{P}$, for example, and have used the $\mathrm{BR} \rightarrow \mathrm{K}$ 
value as a placeholder.) This situation has arisen, no doubt, due to: (1) variations in wild BR, (2) the availability of numerous genetically engineered BR mutants and (3) shifts in values due to environmental conditions.

Time constants are parameters that are easily modified in the model. Thus we believe that we have developed a good modeling technique, and we look forward to creating better, updated models for the various strains of BR as new data become available.

In our follow-on work we are modeling the read cycle by incorporating photodetector and amplifier models into the SPICE circuitry. We then plan to expand the model to multiple voxels in a cube arrangement, simulate the 3-D memory operation, and use the results to design a live demo using a cuvette of BR material. As our knowledge advances, we will build models of other bio-computational elements as well.

\section{REFERENCES}

[1] Nagel, L.W., SPICE2: A Computer Program to Simulate Semiconductor Circuits. Memorandum No. UCB/ERL M520. Electronics Research Laboratory, College of Engineering, University of California, Berkeley. May 9, 1985.

[2] May, J.M., and Mikulecky, D.C., "The simple model of adipocyte hexose transport," Journal Of Biological Chemistry, 2588, No. 19 , October 10, 1982. pp. 11601-11608.

[3] May, J.M., and Mikulecky, D.C., "Glucose utilization in rat adipocytes," Journal Of Biological Chemistry, 258, No. 8, April 25, 1983. pp. 4881-4888.
[4] Seither, R.L., Trent, D.L., Mikulecky, D.C., Rape, T.J, and Goldman, D.I., "Folate-pool interconversions and inhibition of biosynthetic processes after exposure of L1210 leukemia cells to antifolates," Journal Of Biological Chemistry, 264, No. 29, October 15, 1989. pp. 4112-4118.

[5] Seither, R.L., Hearne, D., Trent, D., Mikulecky, D.C., and Goldman, D.I., "SPICE2 network simulation of antifolate effects on purine and pyrimidine biosynthesis: Exploring the role of tetrahydrofolate cofactor depletion versus dihydrofolate feedback inhibition," Computera and Mathematics with Applications, 20, No. 4-6, 1990. pp. 88-101.

[6] Seither, R.L., Trent, D.L., Mikulecky, D.C., Rape, T.J, and Goldman, D.I., "Effect of direct suppression of thymidylate synthase at the 5,10methylenetetrahydrofolate binding site on the interconversion of tetrahydrofolate cofactors to dihydrofolate by antifolates," Journal of Biological Chemistry, 266, No. 8, March 5, 1991. pp. 18016-18023.

[7] Amos, Martyn, Cellular Computing. Oxford University Press, 2004

[8] Schmidt, E.J., Stuart, J.A., Singh, D. and Birge, R.R., "Bacteriorhodopsin-based volumetric optical memory," International NonVolatile Memory Technology Conference. 1998. pp. 84-90.

[9] Ytterdal, T, Fjeldly, T.A., Baier, S., Deng, J., and Shur, M.S., "Modeling and simulation of optoelectronic interconnect systems using a single kernel simulator," Fourth IEEE International Caracas Conference on Devices, Circuits and Systems, Aruba, April 17-19, 2002

[10] Deng, J., Shur, M.S., Fjeldly, T.A., BAIER, S., and Ytterdal, T., "SPICE modeling for mixed electronic/photonic VLSI", Proc. Techcom 2000, Semiconductor Research Corporation (Pub P000740), Phoenix, Arizona. Sept. (2000).

[11] Brower, A.J., Ewing, R.L., Brower, R.W., and Abdel-Aty-Zohdy, H., "SPICE for modeling biological networks," IEEE Midwest Symposium for Circuits and Systems. 2005. pp. 491-494.

[12] http://privatewww.essex.ac.uk/ mpthak/VCSEL\%20SPICE\%20Mode $1 \% 20$ Honeywell.pdf 